\title{
Nanoscale Analysis of Humidity Dependent Tonal Appearance of Platinum/Palladium Prints
}

\author{
Keana $\operatorname{Scott}^{1}$, Matthew L. Clarke ${ }^{2,3}$, and Alline Myers ${ }^{1}$ \\ ${ }^{1}$ National Institute of Standards and Technology, Gaithersburg, MD \\ ${ }^{2}$ Smithsonian Institution, Freer Gallery of Art and Arthur M. Sackler Gallery, Washington, DC \\ ${ }^{3}$ National Gallery of Art, Washington, DC
}

Platinum and palladium photographic prints are prized for their long and nuanced tonal range. Commercial platinum print papers were produced from the 1870 s and continued to the 1930 s. The printing practice continues today with self-prepared papers coated with the sensitive salts and often combining platinum and palladium metals. Many factors can influence the final appearance of the print, including the paper base, the metals used, the developer and the moisture content of the paper during exposure. While the importance of relative humidity $(\mathrm{RH})$ on the printing process was long recognized, the structural changes in the printed nanoparticles that cause this change in appearance had not been observed.

Platinum/palladium $(\mathrm{Pt} / \mathrm{Pd})$ prints were prepared using the modern ammonium process described by Ware.[1] A sensitizing solution mixed to a molar ratio c.a. $4 \mathrm{Fe}: 1 \mathrm{Pt}$ : $1 \mathrm{Pd}$ was coated onto 100\% cotton alum-rosin-starch sized paper. The paper was then split into three sections and equilibrated to three $\mathrm{RH}$ conditions. The three sections were placed in a printing frame with Stouffer $^{1} 21$-step tablet negative and contact printed using a UV sunlamp. Prints were developed by gentle exposure to water vapor and cleared in a three-step bath consisting of 5\% (w/v) disodium EDTA, 2\% sodium sulfite, 5\% tetrasodium EDTA, and then thoroughly washed in running water. After processing the prints exhibit a difference in tone with the print conditioned to low RH giving sepia (warm brown) tones and the print from high $\mathrm{RH}$ giving neutral gray tones. XRF confirms that the relative amounts of platinum and palladium were consistent among the three prints.

FEI Helios 650 NanoLab focused ion beam scanning electron microscope (FIB SEM) equipped with an EDAX Octane Plus silicon drift detector was used to analyze the print surface and prepare the transmission electron microscopy (TEM) specimens of the print paper for the scanning TEM (S/TEM) analysis. The initial surface analysis of the print samples showed the presence of bead-like Pt/Pd particles in all three $\mathrm{RH}$ samples. Figure 1a shows a cluster of these $\mathrm{Pt} / \mathrm{Pd}$ surface particles. Although the overall size distributions of the surface particles were similar across the three samples, the number of the particles increased with the increasing RH. Cross-sectional analysis of the print samples showed that these particles are only present on the print surface. However, the cross-sectional analysis also revealed that there were numerous nanoscale particles throughout the paper fiber and their size and concentration varied with different RH. TEM specimens were prepared from the print samples using a protocol suited for organic materials.[2] To avoid Pt contamination from the gas injection system, TEM specimen preparation was performed using carbon deposition only. Figure 1b shows a typical TEM specimen made from the print

\footnotetext{
${ }^{1}$ Certain commercial entities, equipment or materials may be identified in this document in order to describe an experimental procedure or concept adequately. Such identification is not intended to imply recommendation or endorsement by the National Institute of Standards and Technology, nor is it intended to imply that the entities, materials or equipment are necessarily the best available for the purpose.
} 
samples. Larger $\mathrm{Pt} / \mathrm{Pd}$ particles are localized to the print surface while the nanoscale particles are distributed throughout the paper fiber.

S/TEM energy dispersive x-ray spectrometry (EDS) analysis was performed using FEI Titan 80-300 scanning transmission electron microscope equipped with a Gatan Orius 830 CCD camera, operating at $300 \mathrm{kV}$. S/TEM images clearly indicated that the nanoscale particles observed within the paper fiber are actually agglomerates of smaller nanoparticles where the primary particle size is roughly $5 \mathrm{~nm}$. The main differences between the samples were that, as the RH increased, the agglomerate size increased and the number of agglomerates decreased (Figure 2). EDS analysis of these nanoparticles showed both Pt and Pd signals in all three RH samples.

\section{References:}

[1] Ware, Mike. "An Investigation of Platinum and Palladium Printing." Journal of Photographic Science, vol. 34 (1986): 165-177.

[2] Scott, K. and Giannuzzi, L.A. NIST Special Publication (2015), http://dx.doi.org/10.6028/NIST.SP.1200-16.

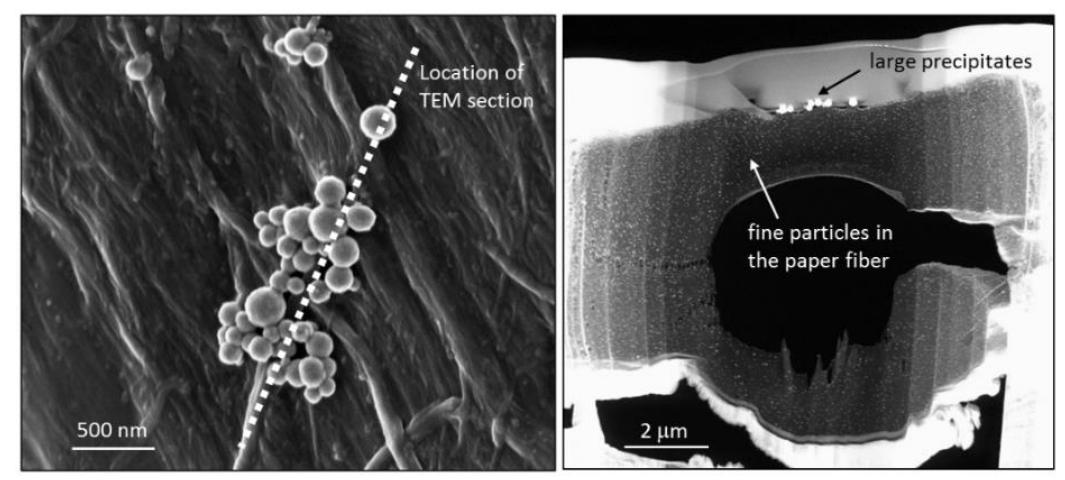

Figure 1. FIB preparation of a TEM specimen of a Pt/Pd print. (A) Print sample surface showing a cluster of $\mathrm{Pt} / \mathrm{Pd}$ surface particles. TEM section was made along the dashed line to include these surface particles and the underlying paper fiber. (B) TEM specimen showing the surface particles and the nanoscale particles dispersed throughout the paper fiber.
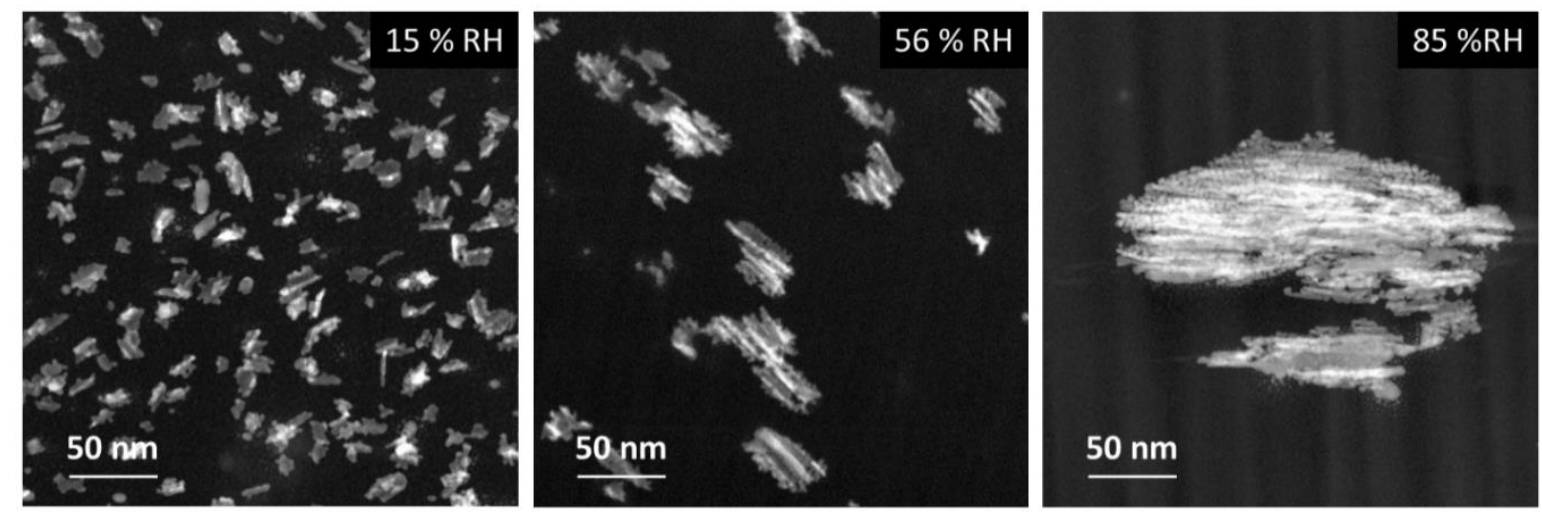

Figure 2. SITEM images that indicate the size of the particulates formed within the cellulose fiber is dependent on the RH during exposure. At low RH there is a high density of small particles. At high RH larger particulates are observed. 